\title{
Longer Versus Shorter Daily Constraint-Induced Movement Therapy of Chronic Hemiparesis: An Exploratory Study
}

\author{
Annette Sterr, PhD, Thomas Elbert, PhD, Irina Berthold, DiplPsych, Sabine Kölbel, DiplPsych, \\ Brigitte Rockstroh, PhD, Edward Taub, PhD
}

ABSTRACT. Sterr A, Elbert T, Berthold I, Kölbel S, Rockstroh B, Taub E. Longer versus shorter daily constraintinduced movement therapy of chronic hemiparesis: an exploratory study. Arch Phys Med Rehabil 2002;83:1374-7.

Objective: To evaluate and compare the effects of 3-hour versus 6-hour daily training sessions in constraint-induced movement therapy (CIMT).

Design: Intervention study, 2-group randomized trial; baseline, pretreatment, and posttreatment measures; 1 -month follow-up (weekly measures).

Setting: University department of psychology in Germany.

Participants: A convenience sample of 15 adults with chronic hemiparesis (13 stroke, 2 traumatic brain injury).

Intervention: CIMT (14 consecutive days; constraint of unaffected hand for a target of $90 \%$ of waking hours) with either 6 hours ( $6 \mathrm{~h} / \mathrm{d}$ group, $n=7$ ) or 3 hours ( $3 \mathrm{~h} / \mathrm{d}$ group, $n=8$ ) of shaping training with the affected hand per day.

Main Outcome Measures: The Motor Activity Log and Wolf Motor Function Test.

Results: Significant improvements in motor function in the laboratory and increased use of the affected hand in the realworld environment were found in both groups. The beneficial effects were significantly greater in the $6 \mathrm{~h} / \mathrm{d}$ group than in the $3 \mathrm{~h} / \mathrm{d}$ group.

Conclusion: The 3-hour CIMT training schedule significantly improved motor function in chronic hemiparesis, but it was less effective than the 6-hour training schedule.

Key Words: Arm; Motor activity; Physical therapy; Rehabilitation; Stroke.

(C) 2002 by the American Congress of Rehabilitation Medicine and the American Academy of Physical Medicine and Rehabilitation

$\mathbf{H}$ EMIPARESIS IS AMONG the most common deficits after stroke, leading in many cases to disability and permanent dependency on community care. In Germany, as well as other industrialized countries, various physiotherapeutic treatments are applied (and paid for by health care services) to improve chronic hemiparesis. However, controlled evaluation studies indicate that the effectiveness of these treatments is minor or moderate at best. ${ }^{1}$ This finding is especially true for

From the Department of Psychology, University of Liverpool, UK (Sterr); Department of Psychology, University of Konstanz, Germany (Elbert, Berthold, Rockstroh); Hegau Jugendwerk, Gailingen, Germany (Kölbel); and Department of Psychology, University of Alabama and Birmingham VA Medical Center, Birmingham, AL
(Taub).

Supported by the German Research Foundation and the US Veterans Administration (grant no. LRO W 98 0410)

No commercial party having a direct financial interest in the results of the research supporting this article has or will confer a benefit upon the author(s) or upon any organization with which the author(s) is/are associated.

Reprint requests to Annette Sterr, PhD, Dept of Psychology, University of Liverpool, Eleanor Rathbone Bldg, Bedford St S, Liverpool, L69 7ZA, UK,
e-mail: asterr@liverpool.ac.uk.

$0003-9993 / 02 / 8310-6925 \$ 35.00 / 0$

doi:10.1053/apmr.2002.35108 the transfer of therapeutic effects into the home environment (real-world outcome). ${ }^{1,2}$ Recently, alternative approaches, which use repetitive training or forced-use procedures, have been applied with increasing success. ${ }^{3-9}$ Constraint-induced movement therapy (CIMT), developed by Taub et al, ${ }^{10}$ is the most effective intervention among these newer methods. Controlled experiments have shown that CIMT can greatly improve the amount of use of the affected extremity in chronic stroke patients, both in the laboratory and in the real-world environment. ${ }^{10-14}$ Results were replicated in different countries with differing health care systems. ${ }^{15.16}$ Currently, CIMT is the subject of a multisite national clinical trial in the United States.

Part of the theoretical framework for CIMT is provided by neurophysiologic and behavioral studies of learned nonuse. ${ }^{17}$ This technique involves 3 key principles: (1) the forced use of the affected hand by restraining the intact arm, (2) training by shaping movements with the affected hand, and (3) massing the practice of both elements. Thus, during 10 consecutive weekdays ( 2 wk), the patient wears a splint and armsling ensemble on the intact arm during waking hours to force the use of the affected hand. In addition, patients are trained for 6 hours a day to perform increasingly complex movements with the affected hand. Applied in this manner, CIMT achieves long-term improvements ( $2 y$ follow-up) of the amount of use and the quality of movement of the affected arm (for reviews, see Taub et a1 ${ }^{12,18}$ )

Because stroke patients with poorer physical condition have less capacity for demanding activities, a 6-hour a day training schedule may be too strenuous for them. The demanding nature of behavioral intervention techniques can be a major concern in stroke patients; it may also act against the therapy's effectiveness, when a patient is pushed beyond his/her endurance limits and becomes fatigued. Studying the effects of enrichment on recovery from brain lesions in animals, Will et al ${ }^{19}$ found that enrichment of 2 hours a day was as beneficial as 24 hours a day. Thus, the question arises: What might the optimal amount of training be? The present study addresses this issue in that it compares the efficacy of the standard CIMT protocol, as used by Taub et al,12 Miltner et al,15 and Kunkel et al, ${ }^{16}$ with a modified version in which the daily training schedule has been reduced by $50 \%$ to 3 hours a day. The study was designed to answer the following questions: (1) Can we replicate the therapeutic efficacy of the standard 6-hour a day protocol found in earlier work? (2) Do patients also improve when treated with 3 hours of daily shaping training? and (3) Are there any differences in the outcome of the 2 treatment schedules?

\section{METHODS}

\section{Recruitment}

A semistructured telephone interview was used to select potentially suitable subjects (first contact). Clients who reported that they had some residual movement of the affected arm were invited to a laboratory for a screening evaluation (second contact). Informed consent was obtained at this time. Patients were accepted for participation in the study after the 
foliowing criteria were met: (1) capability of extending the afíected hand against gravity (wrist: $20^{\circ}$, fingers: $10^{\circ}$ ), (2) minimal balance problems, (3) only minor spasticity of the affected hand, (4) no receptive aphasia (Aachen Aphasia Test $^{20}$ ), (5) a Mini-Mental State Examination ${ }^{21}$ score greater than 20, and (6) a poststroke interval greater than 12 months. The therapy was scheduled so that the baseline interval between second contact and the start of therapy was at least 2 weeks. In the week before their CIMT began, each patient was seen by a neurologist to determine whether his/her general condition was sufficiently good to permit participation. Two patients were deselected from participation on the basis of this examination.

\section{Patients}

Eighteen hemiparetic patients were accepted for the present study. Two subjects were excluded from data analysis because their follow-up measurements were missing. A third patient became ill during the second week of treatment, and that patient's data were also excluded from analysis.

The remaining 15 patients (age range, 23-77y) were randomly assigned to 1 of 2 training groups: the $3 \mathrm{~h} / \mathrm{d}$ group $(n=8$; 2 women; mean age, $68.4 \pm 7.0 \mathrm{y} ; 5$ right hemiparesis) or the $6 \mathrm{~h} / \mathrm{d}$ group $(\mathrm{n}=7 ; 2$ women; mean age, $49.9 \pm 18.5 \mathrm{y} ; 6$ right hemiparesis). Hemiparesis originated from a stroke in 13 participants. In 2 patients, a moderate traumatic brain injury (TBD) had caused the hemiparesis. The neurologic damage occurred $4.8 \pm 4.7$ years (range, $1-17 y$ ) before assignment to the present study. All patients were right-hand dominant before the insult (Edinburgh Inventory ${ }^{22}$ ).

All patients had received extensive physiotherapy during acute and subacute inpatient rehabilitation beginning shortly after the insult, and they had participated in other in- or outpatient rehabilitation programs to a varying degree. For all participants, the last rehabilitation inpatient stay had ended at least 6 months before CIMT.

\section{Treatment}

Participants in the $6 \mathrm{~h} / \mathrm{d}$ group received the standard CIMT protocol with constraint of the unaffected arm for a target of $90 \%$ of waking hours and 6 hours of daily training by using a shaping procedure. ${ }^{10}$ In the $3 \mathrm{~h} / \mathrm{d}$ group, the shaping was reduced to 3 hours a day. Other than the number of hours of daily training, the CIMT protocol was identical for both groups. Treatment was given every weekday for a period of 2 weeks. In addition, constraining the unaffected hand during waking hours promoted the use of the affected hand in the laboratory and at home. On the weekend, only the constraint was worn; no training was provided. Two types of constraints were used: a resting hand splint and armsling ensemble or a specially designed half glove. In patients without balance problems, the splint and sling ensemble was worn on the unaffected hand. In patients with balance problems, a half glove was used to enable the subject to use the unaffected hand for safety in the event of a fall. Patients were assigned to groups according to a pseudorandom order that counterbalanced type of constraint $(3 \mathrm{~h} / \mathrm{d}$ group: 4 sling and 4 half glove; $6 \mathrm{~h} / \mathrm{d}$ group: 4 sling and 3 half glove).

\section{Outcome Measures}

Before they started their CIMT treatment, patients received a medical and neurologic examination. Before and after the intervention period, 2 tests were administered, the Motor Activity Log (MAL) and the Wolf Motor Function Test ${ }^{11}$ (WMFT, modified version ${ }^{10}$ ). The MAL is a 20 -item question- naire that asks how often (amount of use [AOU]) and how well (quality of movement [QOM]) the affected hand was used for 20 activities of daily living (ADLs; eg, cutting bread, carrying a bag). In the WMFT, 16 tasks had to be performed, ranging from gross arm to fine finger movements. For each task, the speed of performance was recorded, with 120 seconds being the maximum time allowed for performance of each item. The performance at each test administration was characterized by median performance time and mean AOU and QOM. ${ }^{10}$ The MAL was administered 7 times: at baseline (second contact), pretreatment (the Friday before the first treatment day [usually Monday]), posttreatment (Monday after the last treatment day), and at follow-up weekly for the next 4 weeks. The WMFT was given twice, before and after the 2-week CIMT period.

The MAL items were subdivided into 2 subcategories: items in which the AOU and QOM of the affected arm was rated $(N=15)$ and items in which the unaffected arm was rated $(\mathrm{N}=5)$. Because unaffected arm use should not change substantially with CIMT, the unaffected arm score served as a type of control or index for comparison. Three-way analysis of variance (ANOVA), incorporating the repeated-measurement factors time (baseline, pre, post, follow-ups 1-4) and the 2 between-group factors training $(3 \mathrm{~h} / \mathrm{d}, 6 \mathrm{~h} / \mathrm{d})$ and constraint (glove vs splint and sling ensemble), were calculated for the 2 MAL scales (AOU, QOM), respectively. WMFT data were analyzed with a 4-way ANOVA comprising the between-group factors training and constraint, and the 2 repeated-measurement factors time (pre, post) and hand (affected, unaffected). We used $t$ tests and ANOVAs of subsets of data for mean comparisons and post hoc analyses.

\section{RESULTS}

The AOU and QOM scores for baseline, posttreatment, and follow-up are plotted separately for the 2 groups in figure 1. Before treatment, mean scores did not differ significantly between groups. After treatment, the AOU and the QOM scores increased substantially in both groups and remained well above the baseline level in the follow-up period in each patient. Across the 4 follow-up measurements, performance was stable for all participants.

The ANOVA for the AOU and QOM parameters reflected the treatment gain by a strong time effect (AOU: $F_{6,66}=62.2$, $P<.01$; QOM: $F_{6,66}=66.8, P<.01$ ). In both parameters, the treatment effect was greater for the $6 \mathrm{~h} / \mathrm{d}$ group than for the $3 \mathrm{~h} / \mathrm{d}$ training group, as indicated by the training by time interaction (AOU: $\mathrm{F}_{6,66}=2.8, P<.05 ;$ QOM: $\mathrm{F}_{6,66}=2.6, P<.05$ ). The difference in motor improvement between the 2 training conditions was particularly prominent, when only the last follow-up was compared with baseline values $\left(\mathrm{F}_{1,11}=9.4, P=.01\right.$; combined AOU and QOM score). In the $6 \mathrm{~h} / \mathrm{d}$ group, the difference between baseline and the 4 follow-up scores comprised 2.0 MAL units for the AOU and 1.6 MAL units for the QOM (maximal score in both measures, 5 units). In the $3 \mathrm{~h} / \mathrm{d}$ group, an increase of 1.2 MAL units was found for the AOU and the QOM. The scores for the unaffected hand did not change with therapy.

For the WMFT, there were significant effects of time $\left(F_{1,11}=12.1, P<.01\right)$ and time by hand $\left(F_{1,11}=8.9, P<.05\right)$, indicating that affected hand performance increased in speed from pre- to posttreatment. The median pre-post performance time gain was 2.34 seconds for the $6 \mathrm{~h} / \mathrm{d}$ group but only .64 seconds for the $3 \mathrm{~h} / \mathrm{d}$ group; the interaction, however, was not significant. When $t$ tests were computed for subsets of the data, the improved performance reached significance in the $6 \mathrm{~h} / \mathrm{d}$ group ( $t=3.5, P<.01$; mean performance time gain, $2.32 \mathrm{~s}$ ). In the $3 \mathrm{~h} / \mathrm{d}$ group, significance was achieved only for the 1-tailed 

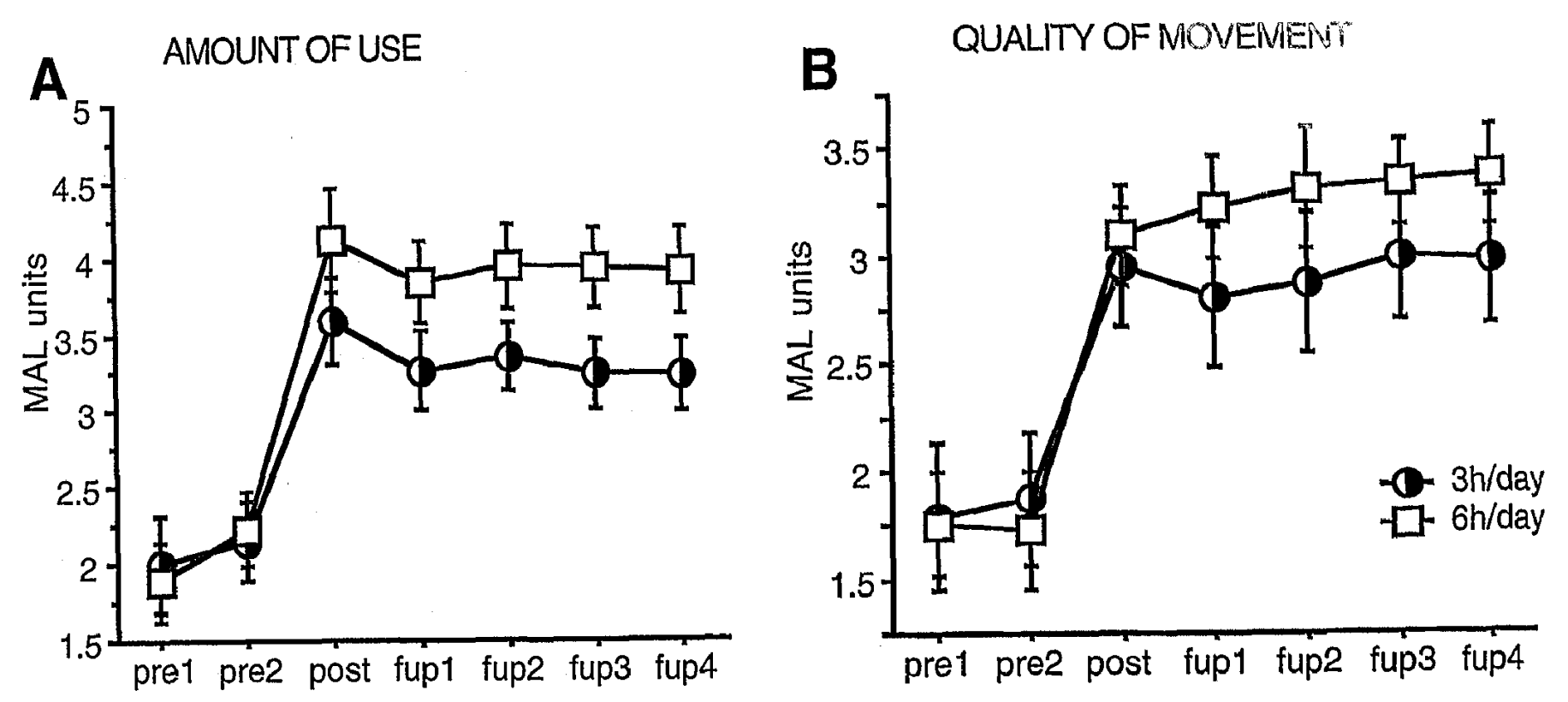

Fig 1. MAL scores and standard errors for (A) amount of use and (B) quality of movement of the affected arm before and after ClMT in patients with chronic upper-limb hemiparesis. The data is given separately for the $6 \mathrm{~h} / \mathrm{d}$ and $3 \mathrm{~h} / \mathrm{d}$ by training groups.

test assumption $(t=2.0, P=.09,2$-tailed; mean performance gain, $1.87 \mathrm{~s})$. No pre-post differences in WMFT performance were found for the unaffected hand.

\section{DISCUSSION}

The present study indicates that CMMT has a pronounced and significant effect on improving the motor ability in chronic hemiplegia. This finding was true for both the standard and the reduced training conditions. Throughout the treatment period, continuous improvement of hand movements in the shaping tasks was observed in each patient. For example, increasingly smaller objects could be picked up faster and with progressively less effort. The patients' functional movement capabilities improved so that new tasks of daily living could be performed outside the laboratory and in the home environment after treatment. The new real-world behaviors that patients performed after treatment consisted of such ADL tasks as eating soup with a spoon, cutting meat, or combing the hair with the affected hand. In line with these observations, statistical analysis of objective outcome measures for the whole sample showed a significant improvement with CIMT in all 3 dependent variables (AOU, QOM, speed of movement). Prepost comparison of WMFT measurements revealed that all patients were able to perform movements with their affected hand faster after therapy. On the MAL, the affected hand performance increased significantly for AOU and QOM. These findings indicate that the affected hand was used more often and with better quality for ADLs; thus, the improvements induced by training in the laboratory transferred into the home environment. Throughout the follow-up period MAL scores for the affected hand dimension remained stable. In contrast, no treatment-related changes were found for the unaffected hand on either the MAL or the WMFT. Because the improvement was specific to the trained hand, the likelihood that nonspecific placebo-type factors could be responsible for the observed treatment effects is low. The present data thus are in full agreement with the following results reported in other CTMT studies 10,12,15,16: (1) chronic hemiparesis after stroke or TBI can effectively be improved by $3 \mathrm{~h} / \mathrm{d}$ or $6 \mathrm{~h} / \mathrm{d}$ CIMT, and (2) func- tionally relevant gains, which increase everyday life performance, are achieved.

Comparing the effectiveness of the $3 \mathrm{~h} / \mathrm{d}$ protocol to that of the standard $6 \mathrm{~h} / \mathrm{d}$ procedure yielded important insight. Statistical analyses of the subgroup data revealed that the $3 \mathrm{~h} / \mathrm{d}$ patients did improve with treatment. However, the therapy effects were stronger in the $6 \mathrm{~h} / \mathrm{d}$ group. Because no pretreatment differences existed in the baseline and pretreatment values between the groups, the greater efficacy of the $6 \mathrm{~h} / \mathrm{d}$ training protocol can be ascribed to the more intense training schedule. It might seem intuitively reasonable that a more intensive training protocol induced larger therapeutic effects. However, patients in both groups wore the restraint during most of their waking hours. The outcome of the present study is relevant to the conceptual framework of CIMT in 2 ways. First, it is not obvious that a reduced CIMT protocol would retain any effectiveness: massed practice is an essential principle in CIMT, and until now little information about the relationship between the amount of training and the effectiveness of the treatment was available. The relationship is probably nonlinear. Thus, the finding that 3 hours of treatment leads to clear, albeit limited, improvement is interesting. Second, the data emphasize the importance of the massing of practice for CIMT. CIMT consists of 3 elements: shaping, the intensity of the training, and the forced use of the affected hand induced by wearing the constraint on the unaffected hand. Little data exist on how these elements interact with regard to treatment efficacy. Because training the patients with the $6 \mathrm{~h} / \mathrm{d}$ schedule is labor intensive and therefore expensive, it is important to know how much training is necessary to achieve maximal efficacy. Our present data show that the effectiveness of the $6 \mathrm{~h} / \mathrm{d}$ training schedule is superior to the $3 \mathrm{~h} / \mathrm{d}$ schedule, although a significant and functionally relevant treatment effect can be obtained with 3 hours of daily training.

In the present study, 4 follow-up measurements were performed over a 1 -month period. Within this period, the treatment effect measured by the MAL remained stable in both groups. For the standard 6h/d CIMT training schedule, longterm follow-up has revealed little or no decline in improve- 
merits up to 2 years after treatment. ${ }^{10,14}$ It is not possible at present to predict long-term retention for the 3-hour group. There might be stability, further improvement up to near the ceiling of the 6-hour group, or a decline, simply because the level of performance reached is insufficient to maintain the use of the affected arm over a prolonged time. Therefore, in addition to the obvious necessity for replication in a larger sample, long-term follow-up data will be necessary to fully ascertain the usefulness of the 3-hour treatment protocol.

\section{CONCLUSION}

The data obtained in the $6 \mathrm{~h} / \mathrm{d}$ training group replicates earlier results $^{18}$ and thus generally confirms the effectiveness of CIMT for the treatment of chronic hemiplegia. The new data from our present study suggests that a reduced 3 -hour training schedule combined with constraint of the unaffected arm significantly improves the patient's movement capabilities and that those capabilities transfer from the therapeutic setting to the home environment. However, the clinical benefits in the $3 \mathrm{~h} / \mathrm{d}$ group are not as great as when 6 hours of daily training are given, indicating greater efficacy of the $6 \mathrm{~h} / \mathrm{d}$ training schedule. One must bear in mind that the present study compared small groups and that future investigations are needed before final conclusions can be drawn.

\section{References}

1. Duncan PW. Synthesis of intervention trials to improve motor recovery following stroke. Top Stroke Rehabil 1997;3:1-20.

2. Emst E. A review of stroke rehabilitation and physiotherapy. Stroke 1990;21:1081-5.

3. Bütefisch C, Hummelsheim H, Denzler P, Mauritz K-H. Repetitive training of isolated movements improves the outcome of motor rehabilitation of the centrally paretic hand. J Neurol Sci 1995;130:59-68.

4. Mauritz $\mathrm{KH}$, Hesse $\mathrm{S}$, Platz T. Late recovery of motor functions. In: Freund H-J, Sabel BA, Witte OW, editors. Brain plasticity: advances in neurology. Philadelphia: Lippincott-Raven; 1997. p 395-408.

5. Peter C, Leidner O. Forced-use-Therpie in der Rehabilitation von Patienten mit Halbseitenlähmung - eine Modifikation für die klinische Praxis. Neurol Rehabil 1997;3:137-44.

6. Ottenbacher KJ, Jannel MS. The results of clinical trials in stroke rehabilitation research. Arch Neurol 1993;50:37-44.

7. Hesse S, Bertelt C, Jahnke MT, et al. Treadmill training with partial body weight support compared with physiotherapy in nonambulatory hemiparetic patients. Stroke 1995;26:976-81.
8. Kriz G, Hermsdörfer J, Marquardt C, Mai N. Feedback-based training of grip force control in patients with brain damage. Arch Phys Med Rehabil 1995;76:653-9.

9. van der Lee JH, Wagenaar RC, Lankhorst GJ, Vogelaar TW, Deville WL, Bouter LK. Forced-use of the upper extremity in chronic stroke patients: results from a single-blind randomized clinical trial. Stroke 1999;30:2369-75.

10. Taub E, Miller NE, Novack TA, et al. Technique to improve chronic motor deficit after stroke. Arch Phys Med Rehabil 1993; 74:347-54.

11. Wolf SL, Lecraw DE, Barton LA, Jann BB. Forced-use of hemiplegic upper extremities to reverse the effect of learned nonuse among chronic stroke and head-injured patients. Exp Neurol 1989; 104:125-32.

12. Taub E, Crago JE, Uswatte G. Constraint-induced movement therapy: a new approach to treatment in physical rehabilitation. Rehabil Psychol 1998;43:152-70.

13. Taub E, Pidikiti RD, DeLuca SC, Crago JE, Effects of motor restriction of an unimpaired upper extremity and training on improving functional tasks and altering brain/behaviors. In: Toole $\mathrm{J}$, editor. Imaging and neurologic rehabilitation. New York: Demos; 1996. p 133-54.

14. Taub E, Uswatte G, Pidikiti R. Constraint-Induced Movement Therapy: a new family of techniques with broad application to physical rehabilitation-a clinical review. J Rehabil Res Dev 1999;36:237-51.

15. Miltner WH, Bauder H, Sommer M, Dettmers C, Taub E. Effects of constraint-induced movement therapy on patients with chronic motor deficits after stroke. Stroke 1999;30:586-92.

16. Kunkel A, Kopp B, Müller G, Villringer K, Taub E, Flor H. Constraint-induced movement therapy for motor recovery in chronic stroke patients. Arch Phys Med Rehabil 1999;80:624-8.

17. Taub E. Somatosensory deafferentation research with monkeys: implications for rehabilitation medicine. In: Ince LP, editor. Behavioral psychology in rehabilitation medicine: clinical applications. New York: Williams \& Wilkins; 1980. p 371-401.

18. Taub $E$, Uswatte $G$, Elbert T. New treatments in neurorehabilitation founded on basic research. Nat Rev Neurosci 2002;3:228-36.

19. Will BE, Rosenzweig MR, Bennett EL, Herbert M, Morimoto HA. A relative brief environmental enrichment aids recovery of learning capacity and alters brain measures after postweaning brain lesions in rats. J Comp Physiol Psychol 1977;91:33-50.

20. Huber W, Poeck K, Weniger D, Willmes $K$. Aachener Aphasie Test (AAT). Göttingen (Germany): Hogrefe Verlag; 1983.

21. Folstein MF, Folstein SE, McHugh PR. "Mini-mental-state." A practical method for grading the cognitive state of patients for the clinician. J Psychiatr Res 1975;12:189-98.

22. Oldfield RC. The assessment and analysis of handedness: the Edinburgh inventory. Neuropsychologia 1971;9:97-113. 